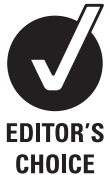

Emergency Department, Manchester Royal Infirmary, Manchester, Lancashire, UK

\section{Correspondence to} Dr Richard Body, Emergency Department, Hope Hospital, Stott Lane, Salford, Manchester M6 8HD, UK;

rbody@doctors.org.uk

Accepted 2 July 2009

\title{
Diagnosing acute myocardial infarction with troponins: how low can you go?
}

\author{
Richard Body, Garry McDowell, Simon Carley, Jamie Ferguson, Kevin Mackway-Jones
}

ABSTRACT

Background Recent consensus guidelines state that acute myocardial infarction (AMI) may be diagnosed in the context of a troponin rise above the 99th percentile of the upper reference limit (URL) with the optimal imprecision of the assay (coefficient of variation, CV) being $\leq 10 \%$. However, at the 99th percentile, modern assays do not have a $\mathrm{CV} \leq 10 \%$.

Objective The authors compared the prognostic implications of placing the diagnostic troponin cut-off at the 99th percentile and at the lowest concentration with a CV $\leq 10 \%$ (functional sensitivity).

Methods The authors prospectively recruited 804 patients presenting to the Emergency Department of a university-affiliated teaching hospital with suspected ACS. All patients underwent $12 \mathrm{~h}$ troponin T testing and were followed up by telephone and chart review.

Outcomes Death or AMI (excluding the index event) and the occurrence of major adverse cardiac events (MACEs) within 6 months.

Results Troponin T elevation below the functional sensitivity predicted the risk of death and AMI (adjusted OR 4.6, $p=0.039$ ) and MACE (adjusted OR 11.10, $p<0.0001$ ) independently of the Thrombolysis in Myocardial Infarction risk score and creatinine levels. Utilising the 99th percentile cut-off, an extra 17 MACEs could be predicted per 1000 patients treated at a cost of identifying 11 patients who would not have developed an event.

Conclusions The results suggest that adopting the lower troponin cut-off would reduce the proportion of 'false negatives' (patients with negative troponin who develop MACE) from $9.6 \%$ to $8.9 \%$. Whether this reduction in 'false negatives' justifies the increase in 'false positives' warrants further investigation and discussion.

\section{BACKGROUND}

According to the 2007 universal definition of acute myocardial infarction (AMI), AMI may be diagnosed in the presence of a rise and/or fall of troponin concentrations with at least one value above the 99th percentile of a healthy reference population, when accompanied by at least one of the following: symptoms of ischaemia, ischaemic ECG changes, development of pathological $\mathrm{Q}$ waves or imaging evidence of myocardial infarction. ${ }^{1}$ Importantly, however, the definition also specifies the optimal level imprecision of troponin assays at the diagnostic cut-off. Imprecision in biochemical assays is evaluated by the coefficient of variation (CV). For diagnosing AMI with cardiac troponins, the CV at the diagnostic cut-off should be $\leq 10 \%$ (a cut-off that is labelled as the 'functional sensitivity' of the assay). Using many contemporary assays, this level of imprecision is only achieved at troponin concentrations above the 99th percentile.

Large-scale research has clearly demonstrated that troponin elevations are markers of adverse prognosis. ${ }^{2}$ Further, the original research into the cardiac troponins demonstrated a risk gradient from the lowest to highest troponin levels. ${ }^{3}$ Troponin elevations below the functional sensitivity of the assay have been shown to be markers of adverse prognosis among apparently healthy 70-year-old men, ${ }^{4}$ among unselected inpatients ${ }^{5}$ and among highly selected groups of patients with confirmed acute coronary syndromes (ACS). ${ }^{6-8}$

Theoretical models suggest that accepting a diagnostic cut-off below the functional sensitivity of the assay will lead to relatively few analytical false-positive troponin results. ${ }^{9}$ Further, in the general population, it has been shown that troponin elevations above the 99th percentile but below the functional sensitivity are rare among subjects without congestive heart failure, left ventricular hypertrophy, chronic kidney disease and diabetes mellitus, adding weight to the suggestion that the 99th percentile cut-off is the more appropriate. ${ }^{10}$

We sought to evaluate the true clinical and prognostic implications of adopting the lower diagnostic troponin cut-off among undifferentiated patients who present to the Emergency Department (ED) with suspected ACS.

\section{METHODS}

This is a substudy of the Early Vascular Markers of Acute Coronary Syndromes (EVaMACS) study, a single-centre prospective diagnostic cohort study. We recruited patients in the ED at Manchester Royal Infirmary, a university-affiliated teaching hospital with an annual ED census of approximately 145000 (comprising approximately 39000 major cases, 43000 minor injuries, 19000 ophthalmological emergencies, 24000 primary care emergencies, 13000 presentations to the Walk in Centre and 7000 others). The study was approved by the Local Research Ethics Committee.

All patients aged $>25$ years who presented to the ED with suspected cardiac chest pain, with the most significant episode occurring within the last $24 \mathrm{~h}$, were eligible for inclusion in the study. We excluded patients if they had another medical condition requiring hospital admission, renal failure needing dialysis, significant chest trauma with suspicion of myocardial contusion, were pregnant, did not speak English or were prisoners. All patients provided written informed consent.

Clinical data were recorded at the time of $\mathrm{ED}$ presentation using a custom-designed clinical report form. The study protocol mandated that all 
patients should have blood taken for troponin $\mathrm{T}$ testing $\geq 12 \mathrm{~h}$ after symptom onset (Roche diagnostics fourth generation assay, 99th percentile $<0.01 \mathrm{ng} / \mathrm{ml}, \mathrm{CV} \leq 10 \%$ at $0.035 \mathrm{ng} / \mathrm{ml}$ ). The timing of serial troponin testing was not dictated by the study protocol and was undertaken at the discretion of the responsible physicians. Patients were followed up after 48 h, 306 days and 6 months by telephone interview and review of hospital records (including clinic letters, investigation reports and details of all hospital admissions and ED attendances, both at Manchester Royal Infirmary and at other centres where appropriate). The National Health Strategic Tracing Service (NSTS) database was also searched for mortality data.

\section{Outcomes}

The primary outcomes of this study were the composites of allcause mortality or AMI (excluding the index event) and the occurrence of any major adverse cardiac events (MACE) within 6 months of initial ED presentation. In accordance with the 2007 universal definition, patients fulfilled the diagnosis of AMI if they had a troponin $\mathrm{T}$ elevation $\geq 0.035 \mathrm{ng} / \mathrm{ml}$ (ie, above the 99th percentile of the upper reference limit with a coefficient of variation $\leq 10 \%$ ) with at least one of the following: symptoms of ischaemia, ECG evidence of AMI (acute ischaemic changes or development of pathological $\mathrm{Q}$ waves) or imaging evidence of new loss of viable myocardium. ${ }^{1} 11$

MACEs were defined as: all-cause mortality; AMI (excluding the index event); the need for urgent coronary revascularisation (UR), as reported by the interventional cardiologist undertaking coronary angiography (this excluded revascularisation following coronary angiography that was undertaken on an elective basis); any unequivocally positive investigation for stress inducible myocardial ischaemia by the time of follow-up (as reported by the physician for the investigation) and a diagnosis of acute left ventricular failure (LVF) requiring hospital treatment. Secondary outcomes included each individual component of the composite primary outcomes and a discharge diagnosis of ACS, as assigned by the responsible physician at the time of hospital discharge.

\section{Statistical methods}

The value of troponin levels for predicting the occurrence of the primary and secondary outcomes was evaluated using logistic regression to calculate ORs and 95\% CI. The effect of potential confounding variables on any observed associations between troponin $\mathrm{T}$ and adverse outcome was analysed by entering troponin $\mathrm{T}$, Thrombolysis in Myocardial Infarction (TIMI) risk score (minus troponin results) and admission creatinine levels as covariates in a multivariate logistic regression model. To aid statistical analysis, troponin $\mathrm{T}$ level was divided into four convenient groups ( $<0.01 \mathrm{ng} / \mathrm{ml}, 0.01-0.03 \mathrm{ng} / \mathrm{ml}, 0.04-0.1 \mathrm{ng} /$ $\mathrm{ml}$ and $>0.1 \mathrm{ng} / \mathrm{ml}$ ) and entered into each of these models as a categorical variable. Sensitivities and specificities of the different troponin cut-offs were calculated together with $95 \%$ CIs. To take account of the paired nature of the measurements, sensitivities and specificities were compared using the McNemar test. Statistical analyses were undertaken in SPSS V.15 (SPSS, Chicago) and MedCalc 9.5.2.0 (MedCalc Software, Mariakerke, Belgium).

\section{RESULTS}

Eight hundred and four patients were recruited to the study between January 2006 and February 2007. Eight patients were excluded because they were found to meet predefined exclusion criteria, meaning that 796 patients were suitable for final analysis. No patients were lost to follow-up. One hundred and fortyeight $(18.6 \%)$ patients were diagnosed as having AMI on their index admission. By 6 months, 19 patients had died (2.4\%), 27 patients had AMI (excluding the index event, 3.4\%), and 154 patients needed urgent coronary revascularisation (19.3\%). In total, 179 patients $(22.9 \%)$ developed an adverse event during 6 months' follow-up. The baseline characteristics of the patients are shown in table 1.

Patients with a troponin $\mathrm{T}$ of $0.01-0.03 \mathrm{ng} / \mathrm{ml}$ were more likely to develop the primary and secondary outcomes within 6 months (figure 1). Relative to patients with troponin $\mathrm{T}$ $<0.01 \mathrm{ng} / \mathrm{ml}$, patients with a troponin T of $0.01-0.03 \mathrm{ng} / \mathrm{ml}$ had an unadjusted OR for the development of death or AMI of 9.23 (95\% CI 2.36 to $36.12, p=0.001$ ) and an OR for MACE of 12.53 (95\% CI 5.23 to $30.04, p<0.0001)$. As shown in figure 2 , patients with troponin T levels between $0.01 \mathrm{ng} / \mathrm{ml}$ and $0.03 \mathrm{ng} / \mathrm{ml}$ were significantly more likely to develop each individual study outcome (with the exception of the development of AMI) than patients with troponin $\mathrm{T}<0.01 \mathrm{ng} / \mathrm{ml}$. When entered into a multivariate model, a troponin T level of $0.01-0.03 \mathrm{ng} / \mathrm{ml}$ was found to predict both primary outcomes independently of the TIMI risk score and serum creatinine levels (table 2). Interestingly, patients with a troponin $\mathrm{T}$ of $0.04-0.1 \mathrm{ng} / \mathrm{ml}$ were more likely to die or have AMI than patients with a troponin $\mathrm{T}$ $>0.1 \mathrm{ng} / \mathrm{ml}$.

The sensitivities and specificities of troponin $T$ at the two proposed diagnostic cut-offs are shown in table 3. Utilising the

Table 1 Baseline characteristics of included patients

\begin{tabular}{|c|c|c|c|c|}
\hline Troponin (ng/ml) & $<0.01$ & $0.01-0.03$ & $0.04-0.1$ & $>0.1$ \\
\hline No $(\%)$ & $625(78.4)$ & $23(2.9)$ & $15(1.9)$ & $133(16.7)$ \\
\hline Age mean $(95 \% \mathrm{Cl})$ & $57.5(56.4$ to 58.6$)$ & $69.0(64.0$ to 74.0$)$ & $63.7(54.7$ to 72.7$)$ & 63.1 (60.8 to 65.3$)$ \\
\hline Sex (percentage male) & 57.9 & 65.2 & 73.3 & 69.9 \\
\hline Prior myocardial infarction (\%) & $151(24.2)$ & $11(47.8)$ & $3(20.0)$ & $29(21.8)$ \\
\hline Previous angina (\%) & $214(34.2)$ & $9(39.1)$ & $3(20.0)$ & $32(24.1)$ \\
\hline Prior coronary intervention (\%) & $135(21.6)$ & $3(13.0)$ & $2(13.3)$ & $20(15.0)$ \\
\hline Previous hypertension (\%) & $309(49.4)$ & $17(73.9)$ & $10(66.7)$ & $63(47.4)$ \\
\hline Previous hyperlipidaemia (\%) & $309(49.4)$ & $13(56.5)$ & 7 (46.7) & $50(37.6)$ \\
\hline Current smoker (\%) & $173(27.7)$ & $5(21.7)$ & $7(46.7)$ & $62(46.6)$ \\
\hline $\begin{array}{l}\text { ECG consistent with myocardial } \\
\text { ischaemia }(\%)\end{array}$ & $112(17.9)$ & $9(39.1)$ & $4(26.7)$ & $101(75.9)$ \\
\hline $\begin{array}{l}\text { ST elevation myocardial infarction } \\
\text { pattern on ECG }(\%)\end{array}$ & $3(0.5)$ & $0(0)$ & $0(0)$ & $57(42.9)$ \\
\hline Creatinine on admission mean $(95 \% \mathrm{Cl})$ & $82(80$ to 84$)$ & 117 (95 to 140$)$ & 87 (70 to 103$)$ & 91 (86 to 97) \\
\hline
\end{tabular}


Figure 1 Troponin T and 6-month outcome. AMI, acute myocardial infarction; MACE, major adverse cardiac event.

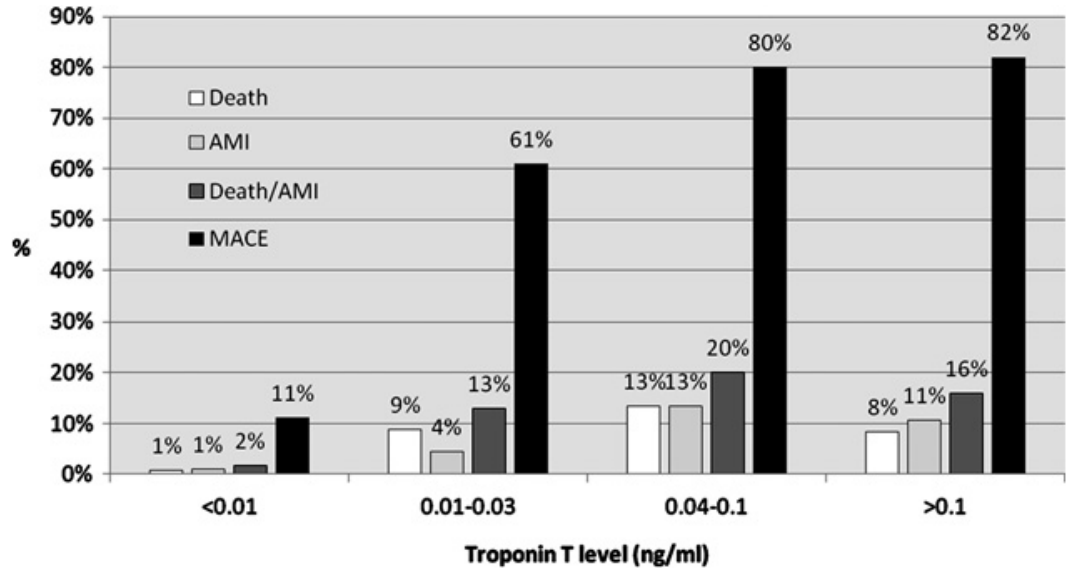

99th percentile cut-off would yield a $6.9 \%$ improvement in sensitivity ( $95 \%$ CI $3.7 \%$ to $6.9 \%, p=0.0001)$ at the cost of a $1.5 \%$ reduction in specificity ( $95 \%$ CI $0.5 \%$ to $1.5 \%, p=0.0039$ ) for identifying patients who would go on to develop a MACE. By accepting the lower cut-off, we would therefore expect that for every 1000 patients treated, an extra 17 MACEs would be predicted at a cost of identifying 11 patients who would not have developed a MACE.

Of the patients with troponin $\mathrm{T} 0.01-0.03 \mathrm{ng} / \mathrm{ml}$, serial sampling was undertaken in 18 (78.3\%) patients. This demonstrated changing levels in $15(65.2 \%)$ patients, although levels did not increase beyond $0.03 \mathrm{ng} / \mathrm{ml}$ before or after $12 \mathrm{~h}$ in any of these patients. In three $(13.0 \%)$ of the patients in this group, troponin $\mathrm{T}$ levels remained unchanged on serial sampling. Five $(21.7 \%)$ of the patients did not undergo serial troponin sampling. Of those patients, one died of cardiac failure, two underwent urgent revascularisation procedures, one was not further investigated, and one had an indeterminate dobutamine stress echocardiogram but did not undergo subsequent investigation.

\section{DISCUSSION}

This study has demonstrated that troponin T elevations below the functional sensitivity of the assay are markers of adverse prognosis in patients with suspected ACS. Elevated but nondiagnostic troponin $\mathrm{T}$ levels independently predicted the development of adverse events at 6 months following adjustment for possible confounding factors. Troponin elevations of this magnitude were found to be significant predictors of all outcomes evaluated with the exception of predicting AMI within
6 months. Accepting the lower diagnostic cut-off would be expected to increase sensitivity for predicting adverse events (potentially identifying 17 at-risk patients per 1000 treated who would not have otherwise been highlighted using troponins). However, the ensuing compromise to specificity might mean that 11 patients would be wrongly identified as 'at risk,' although they would not go on to develop an adverse event.

Interestingly, patients with a troponin $\mathrm{T}$ of $0.04-0.1 \mathrm{ng} / \mathrm{ml}$ were more likely to die or have AMI within 6 months when compared with patients with troponin $\mathrm{T}<0.01 \mathrm{ng} / \mathrm{ml}$. This may be in part explained by the fact that patients with troponin $\mathrm{T}$ between 0.04 and $0.1 \mathrm{ng} / \mathrm{ml}$ are likely to have had non-ST elevation myocardial infarction (NSTEMI), whereas patients with ST elevation myocardial infarction (STEMI) are likely to have higher troponin levels. Previous research suggests that the risk of reinfarction (and consequently of death) is higher in patients with NSTEMI than it is in patients with STEMI. ${ }^{12}$ This phenomenon is perhaps explained by the fact that patients with STEMI have total acute occlusion of a coronary artery with consequent infarction of all of the at-risk downstream myocardium. Patients with NSTEMI may have incomplete coronary occlusion and a substantial amount of viable myocardium still downstream from a vulnerable plaque.

\section{Strengths and limitations}

This study has evaluated and quantified the clinical utility of minimal troponin $\mathrm{T}$ elevations in the precise population for whom the diagnostic test is most widely applied. The main limitation of the study is that the numbers involved were relatively small. Perhaps surprisingly, only 23 patients $(2.9 \%)$ had
Figure 2 Univariate logistic regression analyses demonstrating the odds that patients with troponin $\mathrm{T}$ levels $0.01-0.03 \mathrm{ng} / \mathrm{ml}$ will develop individual outcomes within 6 months (relative to patients with troponin $T$ $<0.01 \mathrm{ng} / \mathrm{ml}$ ). AMl, acute myocardial infarction; LVF, left ventricular failure; MACE, major adverse cardiac event; UR, urgent coronary revascularisation.

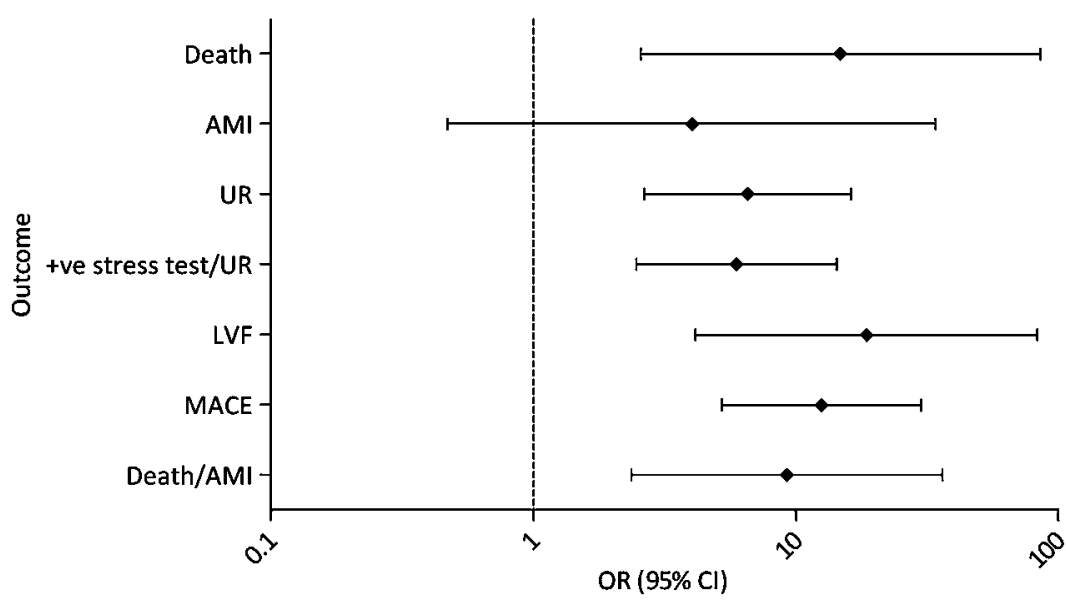


Table 2 Multivariate logistic regression analysis showing the prognostic value of troponin T after adjustment for potential confounding factors

\begin{tabular}{lll}
\hline $\begin{array}{l}\text { Troponin } \\
\mathbf{T}(\mathbf{n g} / \mathbf{m l})\end{array}$ & $\begin{array}{l}\text { Death/acute myocardial } \\
\text { infarction (0R }(\mathbf{9 5 \%} \mathbf{C l}))\end{array}$ & $\begin{array}{l}\text { Major adverse cardiac } \\
\text { event } \mathbf{( 0 R}(\mathbf{9 5} \% \mathbf{C l}))\end{array}$ \\
\hline$<0.01$ & - & - \\
$0.01-0.03$ & $4.60(1.08$ to $19.68, \mathrm{p}=0.039)$ & $11.10(4.44$ to $27.76, \mathrm{p}<0.0001)$ \\
$0.04-0.1$ & $16.43(3.86$ to $69.87, \mathrm{p}=0.0002)$ & $34.22(9.31$ to $125.71, \mathrm{p}<0.0001)$ \\
$>0.1$ & $9.06\left(4.09\right.$ to $\left.20.06, \mathrm{p} 6 \times 10^{-8}\right)$ & $34.16(20.46$ to $57.06, \mathrm{p}<0.0001)$
\end{tabular}

Other variables entered into the model: Thrombolysis in Myocardial Infarction risk score (minus cardiac enzymes); serum creatinine.

troponin elevations below the functional sensitivity of the assay, which limits the capacity for the conclusions to be generalised. The role of this study is therefore best described as hypothesis generation. External validation in larger cohorts of patients is recommended.

Further, we studied the prognostic implications of a single troponin test taken $\geq 12 \mathrm{~h}$ after the onset of the most significant symptoms. While this facilitates a robust, scientific, like-withlike comparison, it does not specifically evaluate the effects of serial troponin testing as advocated for clinical practice by consensus guidelines. ${ }^{1}{ }^{13}$ This should be taken into consideration when evaluating our results and planning future investigations.

\section{Implications of the study}

In recent years, there has been increasing recognition of the need for ongoing risk stratification in troponin-negative patients. A meta-analysis including the results of 19 studies demonstrated that $6.8 \%$ of troponin-negative patients developed death or AMI after a mean of 28 weeks' follow-up. ${ }^{2}$ Accepting a lower troponin cut-off may help to reduce this problem. Indeed, the 2007 consensus guidelines do not undermine the adoption of a troponin cut-off below the functional sensitivity of the assay. ${ }^{1}$

This study adds to the growing body of evidence that troponin elevations below the functional sensitivity are independent markers of adverse prognosis. ${ }^{14}$ The majority of previous work in this area has involved highly selected cohorts of patients who have confirmed ACS, ${ }^{6-8}$ apparently healthy individuals ${ }^{4}$ or unselected hospital inpatients not being investigated for ACS. ${ }^{5}$ This study has investigated the potential clinical implications of accepting a diagnostic cut-off for AMI that is below the functional sensitivity among undifferentiated ED patients with suspected ACS. Our findings are consistent with those of a previous study of $448 \mathrm{ED}$ patients with chest pain, in which patients with troponin I elevations below the functional sensitivity were found to have a significantly worse prognosis than patients with undetectable levels. ${ }^{15}$

Our results suggest that, although patients with troponin elevations below the functional sensitivity are at a lower risk than those with troponin elevations above it, adopting the lower cut-off would reduce the proportion of 'false negatives' (patients who test negative for troponin but develop death or AMI, or need

Table 3 Sensitivity and specificity of troponin T at different cut-offs for identifying patients who would develop adverse events

\begin{tabular}{llll}
\hline Outcome & $\begin{array}{l}\text { Troponin } \\
\text { cut-off } \\
\text { (ng/mI) }\end{array}$ & $\begin{array}{l}\text { Percentage } \\
\text { sensitivity } \\
\text { (95\% CI) }\end{array}$ & $\begin{array}{l}\text { Percentage } \\
\text { sensitivity } \\
\text { (95\% CI) }\end{array}$ \\
\hline Death/acute myocardial & $\geq 0.01$ & $70.0(54.6$ to 81.9$)$ & $81.1(78.1$ to 83.7) \\
infarction & $\geq 0.04$ & $60.0(44.6$ to 73.7$)$ & $83.6(80.8$ to 86.1) \\
Major adverse & $\geq 0.01$ & $66.2(59.2$ to 72.6$)$ & $93.9(91.7$ to 95.7) \\
cardiac event & $\geq 0.04$ & $59.3(52.2$ to 66.1$)$ & $95.4(93.4$ to 97.0) \\
\hline
\end{tabular}

urgent revascularisation within 6 months) from $9.6 \%$ to $8.9 \%$. However, the number of additional 'true positives' would roughly equate to the number of additional 'false positives' identified. Whether this is an acceptable loss of specificity remains unresolved and warrants further investigation and discussion

Our findings also suggest that the overall proportion of patients with suspected ACS who are affected by this issue is relatively small (only $2.9 \%$ of our cohort had troponin elevations below the functional sensitivity). Despite this, the issue remains extremely important for those patients affected, having tremendous medical, economic, psychosocial, medicolegal and ethical implications. While it is without the remit of our study to provide a definitive answer to the problem, we hope that our results will facilitate a more informed debate and inform future large-scale research. In particular, the finding that troponin elevations below the functional sensitivity are associated with significantly worse prognosis will pave the way for future research into high-sensitivity troponin assays with lower limits of detection. Until recently, it has not been possible to precisely quantify the 99th percentile of a healthy reference population because it has been below the lower limit of detection of standard troponin assays. The recent development of high-sensitivity troponin assays has enabled quantification of the true 99th percentile and permits detection of troponin concentrations below that level. Our findings pave the way for further research using high-sensitivity troponin assays to address the issue of whether troponin concentrations below the 99th percentile may also have value for early diagnosis or prognostication of patients with suspected acute coronary syndromes.

Finally, it is important to remember that regardless of the appropriate diagnostic cut-off, AMI is not diagnosed by troponins alone. Judicious use of serial testing and assimilation with all other clinical information are of paramount importance. A positive troponin does not diagnose myocardial infarction; a clinician does

\section{CONCLUSIONS}

Troponin elevations that are above the 99th percentile for a reference population but below the functional sensitivity are independent markers of adverse prognosis in patients with suspected ACS. By lowering the diagnostic cut-off, prognostic stratification was enhanced. Future large-scale research is necessary to further quantify the implications of accepting the lower diagnostic cut-off.

Acknowledgements The authors would like to acknowledge the input of S Roberts who was consulted during both the planning and data-analysis stages with regard to statistical analysis. We would also like to acknowledge the assistance given by all of the nursing, medical and secretarial staff in the Emergency Department, and the support given by the Biochemistry Department at Manchester Royal Infirmary. Finally, we would like to acknowledge the support of the Biomedical Research Centre at Manchester Royal Infirmary.

Funding Central Manchester and Manchester Children's University NHS Trust funded the project. Half of Richard Body's salary was paid by Manchester Metropolitan University during the study period.

\section{Competing interests None.}

Ethics approval Ethics approval was provided by the Local Research Ethics Committee, reference 05/01410/27

Contributors RB had the original idea for this substudy, designed the study protocol recruited and followed up patients, collated and analysed the data, and prepared the manuscript. JF assisted with patient recruitment, data collation and study administration, and reviewed the manuscript. GM, SC and KM-J had the original idea for the study, provided supervision for the project and reviewed the manuscript. 
Patient consent Obtained.

Provenance and peer review Not commissioned; externally peer reviewed.

\section{REFERENCES}

1. Thygesen K, Alpert JS, White HD, et al. on behalf of the Joint ESC/ACCF/AHA/WHF Task Force for the Redefinition of Myocardial Infarction, Biomarker Group, Universal definition of myocardial infarction. Circulation 2007;116:2634-53.

2. Heidenreich PA, Alloggiamento T, Melsop K, et al. The prognostic value of troponin in patients with non-ST elevation acute coronary syndromes: a meta-analysis. J Am Coll Cardiol 2001;38:478-85

3. Antman EM, Tanasijevic MJ, Thompson B, et al. Cardiac-specific troponin I levels to predict the risk of mortality in patients with acute coronary syndromes. N Engl J Med 1996;335:1342-9.

4. Zethelius B, Johnston N, Venge P. Troponin I as a predictor of coronary heart disease and mortality in 70-year-old men: a community-based cohort study. Circulation 2006;113:1071-8

5. Waxman DA, Hecht S, Schappert J, et al. A model for troponin I as a quantitative predictor of in-hospital mortality. J Am Coll Cardiol 2006:48:1755-62.

6. James S, Armstrong P, Califf R, et al. Troponin T levels and risk of 30-day outcomes in patients with the acute coronary syndrome: prospective verification in the GUSTO-IV trial. Am J Med 2003;115:178-84.

7. Morrow DA, Rifai N, Sabatine MS, et al. Evaluation of the AccuTnl cardiac troponin I assay for risk assessment in acute coronary syndromes. Clin Chem 2003:49:1396-8.

8. Hamm CW, Heeschen C, Goldmann B, et al. Benefit of abciximab in patients with refractory unstable angina in relation to serum troponin T levels. N Engl J Med 1999:340:1623-9.
9. Apple FS, Parvin CA, Buechler KF, et al. Validation of the 99th percentile cutoff independent of assay imprecision (CV) for cardiac troponin monitoring for ruling out myocardial infarction. Clin Chem 2005;51:2198-200.

10. Wallace TW, Abdullah SM, Drazner MH, et al. Prevalence and determinants of troponin T elevation in the general population. Circulation 2006;113:1958-65.

11. The Joint European Society of Cardiology/American College of Cardiology Committee. Myocardial infarction redefined-a consensus document of The Joint European Society of Cardiology/American College of Cardiology Committee for the redefinition of myocardial infarction. Eur Heart J 2000;21:1502-13.

12. Montalescot G, Dallongeville J, Van Belle E, et al. STEMI and NSTEMl: are they so different? 1 year outcomes in acute myocardial infarction as defined by the ESC/ACC definition (the OPERA registry). Eur Heart J 2007;28:1409-17.

13. Anderson JL, Adams CD, Antman EM, et al. ACC/AHA 2007 guidelines for the management of patients with unstable angina/non ST-Elevation myocardial infarction: a report of the American College of Cardiology/American Heart Association Task Force on Practice Guidelines (Writing Committee to Revise the 2002 Guidelines for the Management of Patients With Unstable Angina/Non ST-Elevation Myocardial Infarction) developed in collaboration with the American College of Emergency Physicians, the Society for Cardiovascular Angiography and Interventions, and the Society of Thoracic Surgeons endorsed by the American Association of Cardiovascula and Pulmonary Rehabilitation and the Society for Academic Emergency Medicine. J Am Coll Cardiol 2007:50:e1-157.

14. Cook G, Taylor D, France M, et al. Survival among hospital in-patients with troponin T elevation below levels defining myocardial infarction. OJM 2005;98:275-82

15. Kavsak PA, Newman AM, Lustig V, et al. Long-term health outcomes associated with detectable troponin I concentrations. Clin Chem 2007:53:220-7.

\section{Emergency Medicine Questions: Answers}

\section{Theme: Advanced airway}

\author{
From the question on page 265
}

\section{ANSWER 1}

a. True. ${ }^{1}$

b. False. This reliably indicates the tube is in the airway but it may be supra-glottic placement or mainstem bronchus intubation.

c. True. ${ }^{2}$

d. False. It may represent a deflated ETT cuff. Vocalisations in an intubated patient require immediate investigation of cuff inflation and tube placement.

\section{ANSWER 2}

a. False. Atracurium is the preferred intermediate acting nondepolarising muscle relaxant in hepatic failure and renal failure as its elimination is by Hoffman degradation (30\%) and nonspecific ester hydrolysis $(60 \%)$, not renal excretion. ${ }^{3}$

b. True, but generally the preferred management is to mechanically ventilate until paralysis wears off. ${ }^{4}$

c. False. Succinylcholine is more likely to cause bradycardia in children and atropine is generally co-administered to attenuate this effect. However, the likelihood of succinylcholineinduced bradycardia and benefit of atropine have recently been questioned. $^{5}$

d. True. It is reported this is due to up-regulation of receptors. ${ }^{3}$

\section{ANSWER 3}

a. False. The generalised fasciculations suggest adequate motor end plate depolarisation has occurred. Succinylcholine induced masseter spasm is the most likely cause. It is predictive of malignant hyperthermia susceptibility in up to $25 \%$ of cases. ${ }^{6}$

b. True.

c. False. It is generally considered contraindicated in small children aged under 12 years where percutaneous transtracheal ventilation is recommended. ${ }^{8}$

d. False.

Emerg Med J 2010;27:296. doi:10.1136/emj.2010.093047a

\section{REFERENCES}

1. Krauss B, Mason PE. Devices for assessing oxygenation and ventilation. In: Roberts $\mathrm{JR}$, Hedges JR, Custalow CB, et al, eds. Clinical procedures in emergency medicine. 5th edn. Philadelphia, PA: Saunders, 2009.

2. Lang DJ, Wafai Y, Salem MR, et al. Efficacy of the self-inflating bulb in confirming tracheal intubation in the morbidly obese. Anesthesiology 1996;85:246.

3. Caro DA, Laurin EG. Neuromuscular blocking agents. In: Walls M, Murphy MF, Luten $\mathrm{RC}$, eds. Manual of emergency airway management. 3rd edn. Philadelphia, PA Lippincott, Williams \& Wilkins/Wolters Kluwer Health, 2008.

4. Donati F, Bevan DR. Neuromuscular blocking agents. In: Barash PG, Cullen BF, Stoelting RK, eds. Clinical anaesthesia. 5th edn. Philadelphia, PA: Lipincott, Williams \& Wilkins, 2006

5. Hopson LR, Schwartz R. Pharmacological adjuncts to intubation-pre-treatment agents. In: Roberts JR, Hedges JR, Custalow CB, et al, eds. Clinical procedures in emergency medicine. 5th edn. Philadelphia, PA: Saunders, 2009.

6. Naguib M, Lien CA. Pharmacology of muscle relaxants and their antagonists. In: Mille RD, Eriksson LI, Fleisher LA, et al, eds. Miller's anesthesia. 7th edn. Philadelphia, PA: Churchill Livingston, 2009

7. Zhou J, Allen PD, Pessah IN, et al. Neuromuscular disorders and malignant hyperthermia. In: Miller RD, Eriksson LI, Fleisher LA, et al. eds. Miller's anesthesia. 7th edn. Philadelphia, PA: Churchill Livingston, 2009

8. Vissers RJ, Bair AE. Surgical airway management. In: Walls M, Murphy MF, Luten RC eds. Manual of emergency airway management. 3rd edn. Philadelphia, PA: Lippincott, Williams \& Wilkins/Wolters Kluwer Health, 2008. 